\section{Letter in Response to the New SHEA Guideline for Healthcare Workers with Hepatitis B Virus, Hepatitis C Virus, and/or Human Immunodeficiency Virus}

To the Editor-We thank the Society for Healthcare Epidemiology of America (SHEA) for revising the 1997 version of their guideline for the Management of Healthcare Workers Who are Infected with Hepatitis B Virus, Hepatitis C Virus, and/or Human Immunodeficiency Virus ${ }^{1}$ and strongly support the guideline's goal of ensuring the highest level of patient safety. A significant revision of the recommendations was warranted given continued advances in treatment for human immunodeficiency virus (HIV) infection, ${ }^{2}$ the successful implementation of universal precautions, and the lack of any documented cases of HIV transmission from a healthcare worker to a patient in the United States since those attributed to a Florida dentist in $1990 .^{3}$

As the guideline acknowledge, the risk of HIV transmission from healthcare workers to patients is very low. We strongly agree with the general recommendation that HIV-infected healthcare workers who are virologically suppressed should not be barred from performing invasive surgery (referred to as category III in the guideline). However, we disagree with the guideline's approach to implementing this new standard by defining 6 specific criteria under which even well-managed HIV-infected healthcare workers can perform category III procedures, including signing a contract developed in conjunction with an expert review panel. We are concerned that by setting a higher standard for HIV-infected healthcare workers who are successfully maintained on HIV treatment, the revised guideline could unintentionally increase discrimination against these individuals and contribute to the HIVrelated stigma that persists today among healthcare providers and throughout the United States.

In the era of universal precautions, the recommendations unnecessarily create barriers to HIV-infected healthcare workers performing their duties. In the absence of data to support imposing stringent requirements on healthy healthcare workers with HIV infection, the guideline should better reflect current practice standards that have proven effective at preventing healthcare worker-to-patient transmission in many states. We believe that a clarification should be made to the guideline. This clarification should restate that virologically suppressed healthcare workers with HIV infection should be allowed to perform category III procedures, and it should acknowledge that the guideline, as written, is likely difficult to implement and unnecessarily prescriptive given current practice standards. Instead, healthcare workers with HIV infection should be held personally accountable for maintaining their health status, as should healthcare workers with any medical condition. They should be advised to voluntarily seek counsel from their personal physician or an expert review panel on performing specific procedures, as appropriate.

In the absence of this clarification, we are concerned that the new standard could have negative implications for patient safety and harmful consequences for healthcare workers with HIV infection, as summarized below:

- The guideline is overly prescriptive and could unnecessarily raise legal concerns that institutions and health systems may avoid by not hiring healthcare workers with HIV infection.

- The guideline is more restrictive than current policy in many states, despite the effectiveness of present policies in eliminating documented cases of HIV transmission from healthcare worker to patient in the United States. ${ }^{4}$ The new guideline could result in policy changes at the state level brought on by unfounded fears that have already abated.

- An unintended consequence of the guideline could be to discourage healthcare workers from learning their status or, if they know that they are infected with HIV, from disclosing their status to employers.

We thank SHEA for refocusing attention on the important issue of nosocomial infections. However, the emphasis should be on the reinforcement of universal precautions and not on new, unfounded restrictions.

\section{ACKNOWLEDGMENTS}

Potential conflicts of interest. All authors report no conflicts of interest relevant to this article.

Michael S. Saag, MD, FIDSA; Kathleen E. Squires, MD; Judith A. Aberg, MD, FIDSA;

Arlene Bardeguez, MD, MPH, FIDSA; Executive Committee of the HIV Medicine Association (HIVMA)

From the Center for AIDS Research, University of Alabama at Birmingham, Birmingham, Alabama (M.S.S.); Jefferson Medical College, Division of Infectious Diseases, Thomas Jefferson University Hospital, Philadelphia, Pennsylvania (K.E.S.); the Department of Medicine (Infectious Diseases and Immunology), New York University School of Medicine, Bellevue Hospital Center, New York, New York (J.A.A.); and the University of Medicine and Dentistry, New Jersey, New Jersey Medical School, Newark, New Jersey (A.B.).

Address reprint requests to Michael S. Saag, MD, FIDSA, Center for AIDS Research, University of Alabama at Birmingham, 845 19th Street South, BBRB 256, Birmingham, AL 35294-2050 (msaag@uab.edu). Infect Control Hosp Epidemiol 2010; 31(10):1092-1093

(C) 2010 by The Society for Healthcare Epidemiology of America. All rights reserved. 0899-823X/2010/3110-0021\$15.00. DOI: $10.1086 / 656559$ 


\section{REFERENCES}

1. Henderson DK, Dembry L, Fishman NO, et al. SHEA Guideline for Management of Healthcare Workers Who Are Infected with Hepatitis B Virus, Hepatitis C Virus, and/or Human Immunodeficiency Virus. Infect Control Hosp Epidemiol 2010;31:203-232.

2. Walensky RP, Paltiel AD, Losina E, et al. The survival benefits of AIDS treatment in the United States. I Infect Dis 2006;194(1):11-19.

3. Centers for Disease Control and Prevention (CDC). HIV transmission: are patients in a health setting at risk for getting HIV? http://www.cdc .gov/hiv/resources/qa/transmission.htm. Accessed June 8, 2010.

4. The Center for HIV Law \& Policy. Guidelines for hiv-positive health care workers. Published March 2008. http://www.hivlawandpolicy.org/resources/ view/167. Accessed August 18, 2010.

\section{Reply to Saag et al}

To the Editor-On behalf of the Society for Healthcare Epidemiology of America (SHEA), we thank the HIV Medicine Association (HIVMA) for their thoughtful comments ${ }^{1}$ about our recently published guideline for managing healthcare workers (HCWs) infected with bloodborne pathogens. ${ }^{2}$ We agree that the risks of HCW-to-patient transmission of these pathogens are extraordinarily small, but because patient safety is paramount, additional measures-based on specific patient- and disease-related factors-for HCWs infected with bloodborne pathogens may further reduce risks to patient safety. The HIVMA believes that a clarification should be made to the guideline, which should state that virologically suppressed HCWs should be allowed to perform category III procedures and should seek voluntary counsel on specific procedures. To our knowledge, our guideline is the first ever to recommend that HCWs with human immunodeficiency virus (HIV) infection who have their viral burdens suppressed as a result of therapy should be allowed to perform category III procedures. However, we believe that taking certain patient- and disease-related factors into account and partnering with individuals most knowledgeable about the unique aspects of the HCW's work and personal circumstances further minimizes any risk for transmission of bloodborne pathogens. We believe that this partnership among the infected employee, her or his physician, the hospital epidemiology team, and the institution's expert review panel is critical to facilitate optimal management and to assure patient safety. Many institutions are already successfully using this approach in the management of infected HCWs.

Indeed, because transmission of all 3 pathogens-hepatitis B virus (HBV), hepatitis C virus (HCV), and HIV-is rare, we have a very limited scientific foundation on which to construct our guideline. Although we strongly endorse the use of standard precautions for HCWs, we disagree that standard precautions alone are adequate for managing these bloodborne infections in HCWs. In addition, because each infected HCW's circumstance is unique, we strongly favor a case-by-case approach and offer our guideline as a framework for the management of each situation. Guidelines to diminish "significant risk" should be based on the "reasonable judgment of public health officials."

The SHEA guideline provides a contrasting view to the existing US Public Health Service recommendation that "HCWs who are infected with HIV or HBV (and are HBeAg [hepatitis $B$ e antigen] positive) should not perform exposureprone procedures unless they have sought counsel from an expert review panel and been advised under what circumstances, if any, they may continue to perform these procedures. Such circumstances would include notifying prospective patients of the HCW's seropositivity before they undergo exposure-prone invasive procedures." "The SHEA guideline emphasizes that infected HCWs should not be prohibited from participating in patient-care activities solely on the basis of their infection with $\mathrm{HBV}, \mathrm{HCV}$, or HIV and that, unless there is a significant exposure, there should be no requirement to notify patients of a HCW's infection.

As noted in our guideline, because the responsibility for oversight for this guidelines exists at the state level, approaches vary substantially from state to state. Whatever approach is taken by institutions in the successful management of infected HCWs must first be consonant with state and local laws. SHEA views the lack of uniformity of approach in the existing 50-state guidelines as a weakness and has offered our guideline in the hope of creating a framework that will allow a more standardized approach to this complex issue. $^{5}$

The HIVMA letter suggests that the SHEA guideline should be modified to "better reflect current practice standards that have proven effective at preventing healthcare worker-to-patient transmission." Unfortunately, to our knowledge, no published studies document the efficacy of any intervention designed to reduce the risk of HCW-to-patient blood exposure, and certainly no studies demonstrate efficacy in preventing $\mathrm{HCW}$-to-patient transmission. Furthermore, we are not aware of any ongoing program in the United States that is designed to actively detect such cases. Cases are identified only passively and retrospectively. We agree that HCWs, including those infected with these bloodborne pathogens, are personally accountable for maintaining their individual health status. In addition, we offer a constructive framework for these infected HCWs who have certain clinical responsibilities to partner with others and consult with an expert review panel to minimize the risk for transmission of bloodborne pathogens. We believe this partnership is critical to facilitate management and to ensure patient safety. The HIVMA letter also notes that implementing our guideline as written will likely be difficult and unnecessarily prescriptive, given current practice standards. Many institutions and some states have already created the needed infrastructure necessary to manage HCWs infected with bloodborne pathogens in the manner outlined 